\section{LA DESIGNACIÓN TERMINOLÓGICA DE LAS POTENCIAS DE LA INCÓGNITA: ALGUNAS CUESTIONES SOBRE EL TRÁNSITO DEL ÁLGEBRA RETÓRICA AL ÁLGEBRA SINCOPADA EN EL RENACIMIENTO HISPANO}

\author{
Itziar Molina Sangüesa \\ Universidad de Salamanca \\ itziarmolina@usal.es
}

Cómo citar este artículo/Citation: Molina Sangüesa, I. (2016). "La designación terminológica de las potencias de la incógnita: algunas cuestiones sobre el tránsito del álgebra retórica al álgebra sincopada en el Renacimiento hispano". Arbor, 192 (777): a293. doi: http://dx.doi.org/10.3989/ arbor.2016.777n1009

Recibido: 8 abril 2014. Aceptado: 3 noviembre 2015.

RESUMEN: El objetivo de este trabajo es doble: por un lado, estudiar la terminología algebraica referida a las distintas designaciones de las potencias de la incógnita y, por otro, destacar el empleo de ciertas abreviaturas referidas a las mismas, no solo como un mecanismo lingüístico para economizar el discurso, sino por lo que supuso en el desarrollo del álgebra - marcada, desde sus albores, por un estilo puramente retórico - hacia la ciencia eminentemente simbólica en la que se ha erigido. En este proceso evolutivo que va de la palabra al símbolo, la etapa del Renacimiento, caracterizada por la alternancia de los vocablos acuñados por los algebristas italianos frente a los alemanes y por la proliferación de abreviaturas en la notación algebraica (como, por ejemplo, para la expresión de las potencias de la incógnita), es fundamental; motivo por el que revisaremos, analizaremos y estudiaremos estas cuestiones en tres de los tratados matemáticos más relevantes del Quinientos hispano: Libro primero de Arithmética algebrática (1552) de Marco Aurel, Arithmética práctica y speculativa (1562) de Juan Pérez de Moya y Libro de Álgebra en Arithmética y Geometría (1567) de Pedro Núñez Salaciense.

PALABRAS CLAVE: léxico científico-técnico; etimología; abreviaturas (notaciones matemáticas); Álgebra; Renacimiento.

\section{THE TERMINOLOGICAL DESIGNATION OF THE POWERS OF THE UNKNOWN: SOME QUESTIONS ON TRANSIT FROM THE RHETORICAL ALGEBRA TO THE SYNCOPATED ALGEBRA IN HISPANIC RENAISSANCE}

Copyright: (C) 2016 CSIC. Este es un artículo de acceso abierto distribuido bajo los términos de la licencia Creative Commons Attribution-Non Commercial (by-nc) Spain 3.0.

ABSTRACT: This paper has a dual objective: on the one hand, to study the algebraic terminology relating to the different designations of the powers of the unknown and, on the other, to highlight the use of some abbreviations in mathematical language, not only as a linguistic mechanism to economise written discourse, but also for in role in the development of algebra - marked, from its inception, by a purely rhetorical style- into the eminently symbolic science that has emerged. In this evolution from word to symbol, the Renaissance represented an essential stage, characterised by an alternation between the words coined by Italian and German algebraists, and by a proliferation of abbreviations in algebraic notation (for example, for the expression of the powers of the unknown); we therefore review, analyse and study these questions in three of the most important mathematical treatises in sixteenth century Spain: Libro primero de Arithmétic algebrática (1552) by Marco Aurel, Arithmética práctica y speculativa (1562) by Juan Pérez de Moya and Libro de Álgebra en Arithmética y Geometría (1567) by Pedro Núñez Salaciense.

KEYWORDS: scientific-technical lexicon; etymology; abbreviations (mathematical notations); Algebra; Renaissance. 


\section{PRESENTACIÓN}

El objetivo de este estudio es doble: por un lado, estudiar la terminología algebraica referida a las distintas designaciones de las potencias de la incógnita y, por otro, destacar el empleo de ciertas abreviaturas referidas a las mismas, no solo como un mecanismo lingüístico para economizar el discurso, sino por su relevancia en la configuración de una terminología científica que deja constancia del desarrollo y evolución del álgebra en uno de sus tres estadios o periodos: el del álgebra sincopada, característica del Renacimiento.

Para ello centramos el análisis en tres de los tratados matemáticos más relevantes del siglo XVI hispano: Libro primero de Arithmética algebrática (1552) de Marco Aurel ${ }^{1}$, Arithmética práctica y speculativa (1562) de Juan Pérez de Moya ${ }^{2}$ y Libro de Álgebra en Arithmética y Geometría (1567) de Pedro Núñez Salaciense ${ }^{3}$, pertenecientes al corpus del Diccionario de la Ciencia y de la Técnica del Renacimiento (DICTER ${ }^{4}$, editado por Mancho y Quirós (2005).

En primer lugar, examinaremos la etimología de los términos objeto de nuestro estudio: la serie de potencias algebraicas de un número $x$-comprendidas entre la 1a y la 10 10 - , su significado, clasificación y posibles definiciones, que testimoniaremos mediante ejemplos extraídos del corpus del DICTER. A continuación, analizaremos las abreviaturas empleadas por los matemáticos del Quinientos para la expresión de dichas potencias y su empleo en distintos problemas matemáticos, así como los símbolos de adición, sustracción e igualdad que configuran gran parte de las operaciones algebraicas; aspectos de los que, finalmente, extraeremos unas conclusiones.

\section{SOBRE EL ÁLGEBRA}

El álgebra, disciplina que nace como una variante elevada (arte Mayor) o complemento de la aritmética (considerada un arte Menor) - concretamente, de las aritméticas comerciales o calculísticas del S. $\mathrm{XVI}-$, fue desarrollada por griegos, hindúes y otras civilizaciones primitivas - babilonios y egipcios (Couchoud, 1993; Folwer y Robson, 1998). No obstante, se considera que su implantación y desarrollo en Occidente emana del libro escrito por Muhammad ibn Mūsa al-Khwārizmī (Bagdad, ca. 780 -¿ं? 850), titulado Kitab al-Mukhtasar fìhisāb al-jabr w'almuqābala (ca. 825, traducido como Libro conciso de cálculo de restauración y oposición), de donde proviene el nombre de la disciplina: Álgebra (<ár. $a l$-jabr 'restauración'), que en el Renacimiento ${ }^{5}$ con- tó con las variantes: Almucábala (<ár. almuqābala 'oposición') o Regla del álgebra, vulgarmente denominada Regla de la cosa, es decir, la regla o método para resolver y averiguar el valor de la incógnita de un problema dado, que deriva de la traducción del vocablo árabe shay' (con el que se designaba a la cantidad ignota o desconocida) al latín RES 'cosa', y de ahí, finalmente, al italiano còsa, e incluso Regla del cos (que procede de la adaptación del término italiano còsa, al alemán: coss). Tal y como expone Juan Pérez de Moya en el libro séptimo de su Arithmética práctica y speculativa:

Diversos nombres tiene esta regla acerca de varios authores. Unos la llaman Regla de Álgebra, que quiere decir restauratio, o almucábala, que quiere decir opposición o absolución, porque por ella se hazen y absuelven infinitas qüestiones (y las que son impossibles nos las demuestra) assí de Arithmética como de Geometría, como de las demás artes (que dizen) mathemáticas. Otros la nombran Regla de la cosa o [Regla] del cos, porque obrando el nombre bien se le allega. Otros, Reglas reales o Arte mayor. Llámese como cada uno quisiere; su fin no es otro sino mostrar hallar algún número proporcional dudoso demandado (p. 448).

El álgebra desarrollada por al-Khwārizmī, en este primer estadio o periodo, es un álgebra expresada mediante un lenguaje puramente retórico ${ }^{6}$, que evolucionará unos siglos después, de acuerdo con la clasificación establecida por Nesselmann (1842), hacia lo que se conoce como álgebra sincopada en la que, según Etayo Miqueo (1986, p. 147), «se intercalan abreviaturas para hacer más ágil el razonamiento, que sigue expresándose sin embargo en palabras», esto es, momento en el que "el álgebra se escapa de las instrucciones verbales y va hacia las direcciones simbólicas, dejando de ser puramente retórica» (Bell, 2000 , p. 78 ), aspecto que comprobaremos y examinaremos a lo largo de este trabajo.

\section{ANÁLISIS DE SUS DIGNIDADES}

El término dignidad, del latín DIGNITAS 'íd.', según el Diccionario Crítico Etimológico Castellano e Hispánico $(D E C H)$ de Corominas y Pascual, es el nombre que los matemáticos de esta centuria empleaban para referirse a cada una de las potencias de la incógnita, es decir, a cada uno de los "productos que resulta de multiplicar una cantidad o expresión por sí misma una o más veces" (DRAE, 22a edición, s. v. potencia) de una progresión natural en continua proporción. Así lo ponen de manifiesto Aurel y Núñez en el comienzo de sus exposiciones: 
La Regla vulgarmente llamada de la cosa o Arte mayor, [...] en la qual ocorren muchos números de diversos géneros, como quadrados, cúbicos, etc., como en el 9o de Euclides podrás ver. Y para evitar algunos yerros de equivocar un número por otro, quiero poner diez caracteres en una continua proporción y nombrar a cada uno por sí, por su propio nombre que le conviene y pertenesce conforme a su género o dignidad $^{7}$ (Aurel, 1552, fol. 69r).

La primera quantidad d'éstas que llamamos dignidades, que assí van ordenadas en proporción, es la cosa y, por essa causa, le fue dada la unidad por denominación. La segunda es el censo, al qual cupo 2 por denominación. La tercera es el cubo, que tiene 3 por denominación. La quarta es censo de censo, que tiene 4 por denominación. La quinta se llama relato primo, cuya denominación es 5 . La sexta es censo de cubo o cubo de censo y su denominación es 6 . Por este modo proceden los arithméticos y van criando las otras dignidades, y tiene cada una d'ellas la denominación que la orden le da, la qual nos dize quántas proporciones tiene cada una de las dichas quantidades comparada con la unidad, de aquéllas que la cosa guarda con la misma unidad (Núñez, 1567, fol. 24r).

$Y$ en este exemplo pusimos la cosa ser 2 , y conforme a este valor de la cosa veremos el valor de las otras dignidades y cómo suelen ser escriptas: Cosa 2; censo 4; cubo 8; censo de censo 16; relato primo 32; censo de cubo o, cubo de censo 64 . Denominación: $1 ; 2 ; 3 ; 4 ; 5 ; 6$. Las otras dignidades podremos criar por el mismo modo. $Y$ porque esta sciencia no tracta de nombres, bastará nombrarlas por sus denominaciones. Y esto hallaremos en ellas, que toda dignidad multiplicada en sý engendra otra de doblada denominación (Núñez, 1567, fol. 24v).

En estos fragmentos se percibe la gran importancia de emplear un simbolismo eficaz para denotar las distintas potencias de la incógnita:

\subsection{Cosa, lado o raíz}

El término cosa (tomado del italiano còsa, y este del latín CAUSA (DECH) para la traducción del latín RES 'cosa' que, a su vez, es la transliteración del árabe shay'), además de designar la cantidad desconocida o ignota, es decir, la incógnita de una ecuación, se emplea en los tratados matemáticos del Quinientos, en una segunda acepción referida a "cada uno de los valores que puede tener la incógnita de una ecuación" (DRAE, s. v. raíz), en notación simbólica actual: $x$, de cuyo valor atribuido, destaca Moya, depende el del resto de las potencias de la incógnita:
De lo que se ha dicho en estos characteres queda claro que, si la cosa vale 2, el valor de los demás characteres procederá en dupla proporción; y si valiesse la cosa 3 , procederá en tripla, y si 4, en quádrupla, de suerte que, sabido el valor de la cosa, el de los demás characteres es notorio (pp. 451-452).

Además del vocablo cosa, hallamos el nombre raíz ${ }^{9}$ (del latín RĀDIX, -řcis (íd.), DECH) que, entre sus múltiples acepciones, es empleado por los algebristas hispanos como sinónimo de cosa para representar cada uno de los valores que puede tener la $x$, esto es, la incógnita de una ecuación:

Y para evitar algunos yerros de equivocar un número por otro, quiero poner diez caracteres en una continua proporción y nombrar a cada uno por sí, por su propio nombre que le conviene y pertenesce conforme a su género o dignidad, y son los siguientes: el primero se llama dragma o número; el segundo, raýz o cosa; el tercero, censo; el quarto, cubo [...] (Aurel, 1552 , fol. 69r).

Por último, documentamos el sustantivo lado (del latín LĂTŬs, $D E C H$ ) como sinónimo de cosa y raíz:

El co. es raýz o lado de un quadrado equilátero y es el primero de los números de una continua proporción, porque n. es como uno, el qual no es número (Aurel, 1552, fol. 69v).

El segundo se dize cosa. Es raýz o lado de un número quadrado, y éste es el primero de los números de una continua proporción. Su valor es variable, porque, assí como si aviendo de poner algunos números proporcionales puede el primero ser unas vezes una quantidad y otras vezes otra, assí esta cosa no tendrá proprio valor, antes tendrá el que le quisieres dar, assí por enteros como por quebrados (Pérez de Moya, 1562, p. 449),

que refleja las frecuentes imbricaciones entre álgebra y geometría - al igual que sucede con la tercera potencia, designada cubo-, ya desde las matemáticas árabes, pues, emplean para la resolución y planteamiento de ecuaciones construcciones geométricas; influencia directa de los métodos expuestos en los libros de Euclides, que se convirtieron en una referencia constante (Paradis y Malet, 1989, p. 50). Por ejemplo, una de las imágenes de los cuadrados diseñados por Núñez, como la Figura 1, para la resolución de una de las conjugaciones simples ${ }^{10}$, concretamente, la primera: «censos yguales a cosas» (fol. 1r), terminología utilizada por el portugués para referirse a una ecuación de segundo grado del tipo $a x^{2}=b x$ : 
La primera regla, que responde a la primera conjugación, diximos que, si las cosas fueren yguales a censos, partiremos el número de las cosas por el número de los censos y pronunciaremos el número quociente por valor de la cosa, tiene esta razón que agora avemos señalado: que puesto que lo que viene en la partición sea el número de cosas que vale 1 censo y no las unidades que contiene 1 cosa, empero, porque tantas unidades tiene cada 1 cosa quantas cosas tiene el censo, justamente tomaremos el número de cosas que vale 1 censo por el número de unidades que vale 1 cosa:

Figura 1. Conjugación simple (Núñez, 1567, fols. 6r-6v)

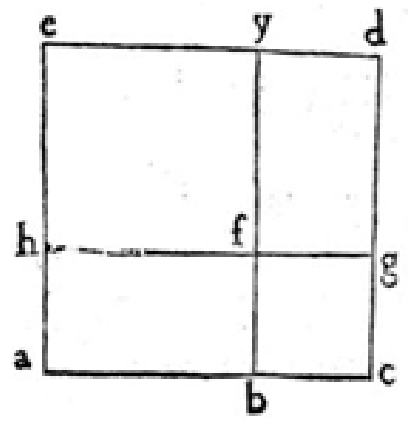

\subsection{Censo}

Tomado del latín CENSUS, -ūs, según el Oxford Latin Dictionary $(O L D)$, es el término acuñado por Gerardo de Cremona ${ }^{11}$ en el siglo XII para la traducción del árabe $m \bar{a}{ }^{12}$ empleado por al-Khwārizmī en su Al-jabr w'almuqābala para la expresión de la segunda potencia de la incógnita, esto es, el cuadrado de la incógnita, $x^{2}$. Este término se documenta en los tratados más relevantes e influyentes del Medievo, como el Liber abacci (1202) de Leonardo de Pisa ${ }^{13}$ o la Suma (1484) de Luca Pacioli ${ }^{14}$.

A pesar de que es censo un término recurrente en gran parte de las aritméticas publicadas a lo largo de los siglos XII-XVII, no aparece recogido, sin embargo, con esta acepción matemática, en ninguno de los repertorios lexicográficos consultados.

Tal y como se deduce de los testimonios del Quinientos, se puede definir censo como la "segunda potencia de un número o expresión algebraica, que se obtiene multiplicando estas cantidades por sí mismas" (en notación simbólica actual: $x^{2}$ ):

El tercero se dize censo. Denota un número quadrado. Procede de la multiplicación de la cosa por sí misma. Como si pones por exemplo que la cosa vale 2 , el censo valdrá 4; y si la cosa vale 3 el censo valdrá 9, y assí procederás en infinito. De lo qual se entiende ser la cosa raýz del censo (Pérez de Moya, 1562, p. 449).

\subsection{Cubo}

Tomado del latín cŭBus y este del gr. кúßoৎ 'cubo', 'dado' $(D E C H)$, es el término empelado para designar la "tercera potencia de un número o expresión algebraica, que se obtiene multiplicando estas cantidades dos veces por sí mismas" (DRAE, 22a edición), que en notación simbólica actual equivale a $x^{3}$, de acuerdo con la explicación de Pérez de Moya:

El quarto se dize cubo. Denota un número cúbico. Procede multiplicando el censo por la cosa, de suerte que si ponemos por exemplo que la cosa vale 5 , a este respecto el censo vale 25 , y el cubo 125 (p. 449).

\subsection{Censo de censo}

El compuesto sintagmático censo de censo, es decir, dos veces censo o cuadrado, es el término empleado por los algebristas para referirse a la "cuarta potencia de un número o expresión algebraica, que se obtiene multiplicando estas cantidades tres veces por sí mismas" (en notación simbólica actual: $x^{4}$ ), como se comprueba en los siguientes ejemplos:

El quinto quiere decir censo de censo. Denota un número que ha sido dos vezes quadrado; quiero dezir que es un número del qual se podrá sacar dos vezes raýz quadrada, assí como 16, que la primera raýz quadrada es 4 , y de 4 la segunda es 2 . Procede de la multiplicación del censo por sí mismo o de la cosa por el cubo. Como si la cosa vale 3 , el censo vale 9 , el cubo 27, y el censo de censo 81 ; este 81 se dize número dos vezes quadrado, por razón que se puede d'él sacar otras tantas vezes raýz quadrada (Pérez de Moya, 1562, p. 450).

La primera quantidad d'éstas que llamamos dignidades, que assí van ordenadas en proporción, es la cosa y, por essa causa, le fue dada la unidad por denominación. La segunda es el censo, al qual cupo 2 por denominación. La tercera es el cubo, que tiene 3 por denominación. La quarta es censo de censo, que tiene 4 por denominación (Núñez, 1562, fol. 24r).

Un aspecto interesante, que detallamos en el apartado 5 , es el empleo de la adición o multiplicación como técnica para la creación de una nueva terminología, en la que las reduplicaciones y yuxtaposiciones sirven como mecanismo para elevar las potencias a términos mayores, en palabras de Núñez (1567, fol. 24r-25r): «por este modo proceden los arithméticos y van criando las otras dignidades», al afirmar que «toda dignidad multiplicada en sý, engendra otra de doblada denominación». 


\subsection{Relato primero, relato primo o sursólido}

Compuesto sintagmático formado por el sustantivo relato (tomado del latín RELĀTUS, -ūs, OLD) y el adjetivo numeral ordinal culto primero o su expresión acortada: primo (del latín PRīmus 'primero', DECH), aparece en las obras de Aurel, Pérez de Moya y Núñez Salaciense para referirse a la "quinta potencia de un número o expresión algebraica, que se obtiene multiplicando estas cantidades cuatro veces por sí mismas" (en notación simbólica actual: $x^{5}$ ):

Procede de la multiplicación del valor de la cosa por el del censo de censo, o el censo por el cubo. Como si la cosa valiesse 2 , el censo valdrá 4 , el cubo 8 , el censo de censo 16, el primero relato 32 (Pérez de Moya, 1562, p. 450).

La primera quantidad d'éstas que llamamos dignidades, que assí van ordenadas en proporción, es la cosa y, por essa causa, le fue dada la unidad por denominación. La segunda es el censo, al qual cupo 2 por denominación. La tercera es el cubo, que tiene 3 por denominación. La quarta es censo de censo, que tiene 4 por denominación. La quinta se llama relato primo, cuya denominación es 5 (Núñez, 1567, fol. 24r).

Este compuesto proviene de la tradición matemática italiana y aparece documentado, entre otras, en la Suma de Pacioli. No obstante, por otro lado, encontramos el nombre sursólido, compuesto del latín sŭRDUM 'sordo' $(D E C H)$ y sŏLĭDus 'íd.' $(D E C H)^{15}$ del que es sinónimo, procedente de la corriente establecida por los algebristas alemanes, como Adam Ries ${ }^{16}$ o Christoph Rudolff ${ }^{17}$; de ahí que se atestigüe, como era de esperar, en el Libro de Álgebra escrito por el germano Aurel y también en la Aritmética de Moya, que sigue fielmente el Álgebra - publicada en 1552- del primero (Cf. Rey Pastor, 1926, p. 105):

Y para evitar algunos yerros de equivocar un número por otro, quiero poner diez caracteres en una continua proporción y nombrar a cada uno por sí, por su propio nombre que le conviene y pertenesce conforme a su género o dignidad, y son los siguientes: el primero se llama dragma o número; el segundo, raýz o cosa; el tercero, censo; el quarto, cubo; el quinto, censo de censo; el sexto, sursolidum o primo relato (Aurel, 1552, fol. 69r).

Del sursolidum o primo relato: El R. es siempre número irracional, porque no tiene raýz quadrada ni raýz cúbica. Procede multiplicando el cce. con la co. o el cu. con el ce.; como si la co. valiesse 2; el ce., 4; el cu., 8; el cce., 16; y R., 32. Es figura prolongada (Aurel, 1552, fol. 69v).
El sexto se dize primero relato o sursolidum. Denota un número que no tiene raýz quadrada ni cúbica; solamente tiene raýz relata, como se declara en el capítulo 3. (Pérez de Moya, 1562, p. 450).

\subsection{Censo de / y cubo, censo cúbico}

El siguiente compuesto sintagmático, formado por los sustantivos censo y cubo, o el adjetivo cúbico (tomado del latín cuBıcus, -A, -UM y este del gr. кußıкós, $O L D)$, hace referencia a la "sexta potencia de un número o expresión algebraica, que se obtiene multiplicando estas cantidades cinco veces por sí mismas" (en notación simbólica actual: $\left.x^{6}\right)$ :

El séptimo se dize censo y cubo. Denota un número quadrado cubicado, o un cubo quadrado; finalmente, es un número del qual se puede sacar raýz quadrada; y de la quadrada raýz cúbica, y al contrario. Assí como 64, del qual la raýz quadrada es 8 , y d'estos 8 la cúbica es 2 ; o de sesenta y quatro la raýz cúbica es 4 , y del 4 la quadrada es 2 . Procede multiplicando el valor de la cosa por el primero relato; o el censo por el censo de censo; o multiplicando el cubo por sí mismo, o cubicando el censo (Pérez de Moya, 1562, p. 450).

Como si la cosa vale dos, el censo valdrá 4 , el cubo 8 , el censo de censo 16 , el primero relato 32 , el censo cúbico 64 (Pérez de Moya, 1562, p. 451).

La primera quantidad d'éstas que llamamos dignidades, que assí van ordenadas en proporción, es la cosa $y$, por essa causa, le fue dada la unidad por denominación. La segunda es el censo, [...]. La sexta es censo de cubo y su denominación es 6 (Núñez, 1567, fol. 24r).

\subsection{Relato segundo o bisursólido}

Similar a la quinta potencia o expresión algebraica, hallamos el compuesto sintagmático formado por el sustantivo de origen latino relato y el adjetivo numeral ordinal segundo que designa la "séptima potencia de un número o expresión algebraica, que se obtiene multiplicando estas cantidades seis veces por sí mismas" (en notación simbólica actual: $x^{7}$ ):

2.187 Dignidad séptima: relato segundo. / 729 Dignidad sexta: censo de cubo. / 243 Dignidad quinta: relato primo. / 81 Dignidad quarta: censo de censo. / 27 Dignidad tercera: cubo. / 9 Dignidad segunda: censo. / 3 Dignidad primera: cosa. (Núñez, 1567, fol. 27r).

Así como el término bisursólido, compuesto por el prefijo latino BI- 'dos veces' y el nombre sursólido, acuñado por los cosistas alemanes: 
Y para evitar algunos yerros de equivocar un número por otro, quiero poner diez caracteres en una continua proporción y nombrar a cada uno por sí, por su propio nombre que le conviene y pertenesce conforme a su género o dignidad, y son los siguientes: el primero se llama dragma o número; el segundo, raýz o cosa; el tercero, censo; el quarto, cubo; el quinto, censo de censo; el sexto, sursolidum o primo relato; el séptimo, censo y cubo; el octavo, bissursolidum o segundo relato (Aurel, 1552, fol. 69r).

El octavo se dize segundo relato o bissursolidum. Es un número de la propriedad que diximos ser el sexto, porque no tiene raýz quadrada ni cúbica. Procede multiplicando el valor de la cosa por el censo y cubo; o el primero relato con censo, o censo de censo por cubo. Y si la cosa vale 2, el segundo relato valdrá 128 (Pérez de Moya, 1562, p. 450).

\subsection{Censo (de) censo de censo}

De nuevo, mediante un mecanismo de redundancia o triplicación, en este caso, del término censo, alcanzamos la "octava potencia de un número o expresión algebraica, que se obtiene multiplicando estas cantidades siete veces por sí mismas" $\left(x^{8}\right)$ :

El nono se dize censo de censo de censo. Denota un número tres vezes quadrado, del qual se podrá sacar otras tantas vezes raýz quadrada. Assí como 256, de los quales la primera raýz quadrada es 16 , la segunda 4 , y d'estos 4 la tercera es dos. Procede multiplicando el valor de la cosa por el segundo relato; o el censo cubo por el censo; o el primero relato con cubo, o multiplicando el censo de censo por sí mismo (Pérez de Moya, 1562, p. 451).

Para demonstración d'esta regla, procederemos continuando la proporción tripla hasta la décima dignidad y aplicaremos nuestra demonstración a un exemplo, como si generalmente demonstrássemos en todos [...]. Dignidad 10: censo de relato primo 59.049. / Dignidad 9: cubo de cubo 19.683. / Dignidad 8: censo de censo de censo 6.561. / Dignidad 7: relato segundo 2.187. / Dignidad 6: censo de cubo 729. / Dignidad 5: relato primo 243. / Dignidad 4: censo de censo 81. / Dignidad 3: cubo 27. / Dignidad 2: censo 9. / Dignidad 1: cosa 3. (Núñez, 1567, fols. 27r-27v).

\subsection{Cubo de cubo}

De manera análoga a la cuarta potencia, censo de censo, testimoniamos el compuesto sintagmático formado por cubo, la preposición de y cubo, es decir, dos veces cubo, que da nombre a la "novena potencia de un número o expresión algebraica, que se obtiene multiplicando estas cantidades ocho veces por sí mismas" (en notación simbólica actual: $x^{9}$ ):

El décimo se dize cubo de cubo. Denota un número dos vezes cubicado, del qual se podrá sacar dos vezes raýz cúbica. Assí como 512, de los quales la primera raýz cúbica es ocho y de ocho es dos. Procede multiplicando la cosa por el censo de censo de censo; o el segundo relato por el censo; o el censo y cubo por cubo; o el primero relato por censo de censo, o cubicando el cubo (Pérez de Moya, 1562, p. 451).

Que este número 19.683 se deva llamar cubo de cubo provaremos assí: el número cubo, multiplicado en sí, haze otro que es censo del mismo cubo. Y este censo de cubo, multiplicado en el mismo cubo, haze el cubo de cubo. Avrá, por tanto, del cubo de cubo a la unidad nueve proporciones, conviene a saber: 3 del cubo a la unidad y tres del censo del cubo al cubo; y otras tres del cubo de cubo al censo de cubo. Por esta causa, el cubo de cubo es la nona dignidad, que en este exemplo, en el qual posimos la cosa valer 3 , terná de valor 19.683 (Núñez, 1567, fols. 28r-28v).

Según la clasificación establecida por Marco Aurel y Pérez de Moya, cubo de cubo es la última de las expresiones algebraicas de una continua proporción:

Y para evitar algunos yerros de equivocar un número por otro, quiero poner diez caracteres en una continua proporción y nombrar a cada uno por sí, por su propio nombre que le conviene y pertenesce conforme a su género o dignidad, y son los siguientes: el primero se llama dragma o número; el segundo, raýz o cosa; el tercero, censo; el quarto, cubo; el quinto, censo de censo; el sexto, sursolidum o primo relato; el séptimo, censo y cubo; el octavo, bissursolidum o segundo relato; el noveno, censo censo de censo; el décimo y último d'ellos es cubo de cubo (fol. 69r).

\subsection{Censo de relato primero}

Finalmente, hallamos el compuesto sintagmático censo de relato primero, que equivale al cuadrado del relato primero o quinta potencia $\left(x^{5}\right)$, es decir, a la "décima potencia de un número o expresión algebraica, que se obtiene multiplicando estas cantidades nueve veces por sí mismas" (en notación simbólica actual: $\left.x^{10}\right)$, documentada únicamente en dos ocasiones, en la obra del matemático portugués Núñez Salaciense:

Para demonstración d'esta regla, procederemos continuando la proporción tripla hasta la décima dignidad y aplicaremos nuestra demonstración a un 
exemplo, como si generalmente demonstrássemos en todos [...]. Dignidad 10: censo de relato primo 59.049. / Dignidad 9: cubo de cubo 19.683. / Dignidad 8: censo de censo de censo 6.561. / Dignidad 7: relato segundo 2.187. / Dignidad 6: censo de cubo 729. / Dignidad 5: relato primo 243. / Dignidad 4: censo de censo 81. /Dignidad 3: cubo 27. / Dignidad 2: censo 9. / Dignidad 1: cosa 3. (fols. 27r-27v).

Y haremos la ygualación multiplicando en cruz, y resultarán 10.000 censos yguales a 100 censos de cubo más 1 censo de relato primo, que es décima dignidad (fol. 206v).

\section{ANÁLISIS DE SUS ABREVIATURAS}

El influyente matemático italiano, Luca Pacioli, no solo en la Suma, sino también en otro de sus más célebres textos, De divina proportione (1509), afirma:

Utilizaremos muchos y diversos caracteres y abreviaturas que se acostumbran a usar en semejantes facultades [...]. Y solo con el fin de evitar una excesiva prolijidad en la escritura, y también en la lectura, ya que de otro modo se llenaría de tinta mucho papel. Del mismo modo, también nosotros en matemáticas, para el álgebra, es decir, la práctica especulativa, usamos otros caracteres que significan cosa, censo y cubo, y los demás términos, tal y como se contiene en nuestra obra [Summa de arithmetica, geometría, proportioni et proportionalitá] (Trad. Calatrava, 1991, pp. 40-41).

En esta misma línea, Marco Aurel (1552, fol. 69r) justifica el empleo de las abreviaturas a lo largo de su tratado: "pónense los caracteres porque son breves y por evitar la prolixidad de escrivir tales nombres a la larga», aunque admite otras posibilidades: «los que aquí porné no es de necessidad por fuerça que éstos y no otros hayan de ser, porque cada uno puede poner los que a él plazerán, o si querrá escrivir los dichos nombres a la larga, podrá hazerlo, pues no haze nada al caso. Yo, al presente, pongo los siguientes»:

Figura 2. Notaciones Marco Aurel (1552, fol. 69r)

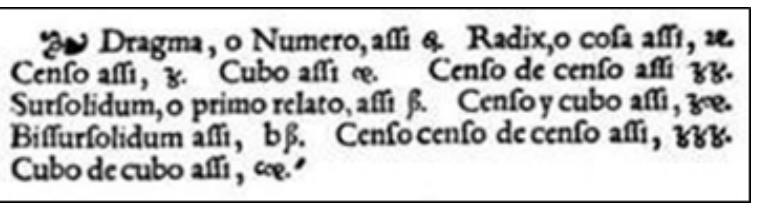

Abreviaturas que transcribimos del siguiente modo: «Dragma o número, assí: $n$. Rádix o cosa, assí: co. Censo, assí: ce. Cubo, assí: cu. Censo de censo, assí: cce. Sursolidum o primo relato, assí: $R$. Censo y cubo, assí: cecu. Bissursolidum, assí: RR. Censo, censo de censo, assí: ccce. Cubo de cubo, assí: ccu.»
A continuación, alude a la proporción a través de la cual se generan potencias cada vez más elevadas:

Ya que has visto la invención, propiedad y significación de los dichos 10 números o caracteres de una continua proporción y como para el presente no pienso serán menester más, no me alargo más. Mas si caso fuere que huviesses menester más de los ya dichos, podrás hallarlos como has visto en la mesma continua proporción, y como por una tabla aquí presente verás, en la qual porné tres exemplos. El primero, en dupla; el segundo, en tripla; el tercero, en quádrupla proporción. Y esto para que puedas ver cómo suben y cómo te has de haver con ellos, quando huviesses menester más de los ya dichos:

Figura 3. Potencias de la incógnita según tipo de proporción. Marco Aurel (1552, fols. 70r-70v)

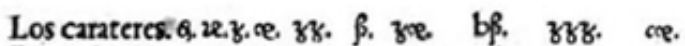 Prop. dupla. 1.2.4 8, 16, 32, 64 128. 256. 512. Prop. tripla. 1.3.9.27.81, 243.729. 2187. 6561. 19683. Prop.çdrupla. 1.4.16.64.256.1024.4096.16384.65536.262144.}

Del mismo modo, Pérez de Moya declara (1562, p. 449):

En este capítulo se ponen algunos characteres, dando a cada uno el nombre y valor que le conviene, los quales son inventados por causa de brevedad. $Y$ es de saber que no es de necessidad que éstos y no otros ayan de ser, porque cada uno puede usar de los que quisiere e inventar muchos más, procediendo con la proporción que le paresciere. Los characteres son éstos:

Figura 4. Notaciones Juan Pérez de Moya (1562, p. 449)

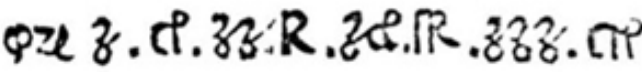

Y en el capítulo tercero del libro dedicado al álgebra reconoce «algunos characteres que yo uso, por no aver en la stampa otros»:

Por los diez characteres que en el precedente capítulo se pusieron uso éstos. Por el que dizen número, n.; por la cosa, co.; por el censo, ce.; por cubo, cu.; por censo de censo, cce.; por el primero relato $R$.; por el censo y cubo, cecu.; por el segundo relato $R R$.; por censo de censo de censo, ccce., por cubo de cubo ccu. (1562, pp. 452-453),

testimonios en los que se deduce que no son convenciones fijadas. No obstante, son abreviaturas asumidas y empleadas con asiduidad y homogeneidad por 
los matemáticos hispanos ${ }^{18}$ para la notación de las sucesivas potencias de la incógnita, de acuerdo con la corriente establecida por la escuela italiana y la influencia ejercida por la Suma de Pacioli, la cual determinó en gran parte, según Paradis y Malet (1989, p. 135), «las notaciones empeladas en Italia, y en los países que culturalmente dependían de ella, hasta prácticamente 1600 ». De hecho, afirman que

la contribución más importante de la Suma son las notaciones. En la obra de Pacioli se da un paso adelante fundamental, desde la simple y pesada retórica de los árabes y del mismo Fibonacci, hacía una simbología específica de las relaciones algebraicas (1989, pp. 135-136).

Figura 5. Notaciones Luca Pacioli (1494, p. 155, Al margen)

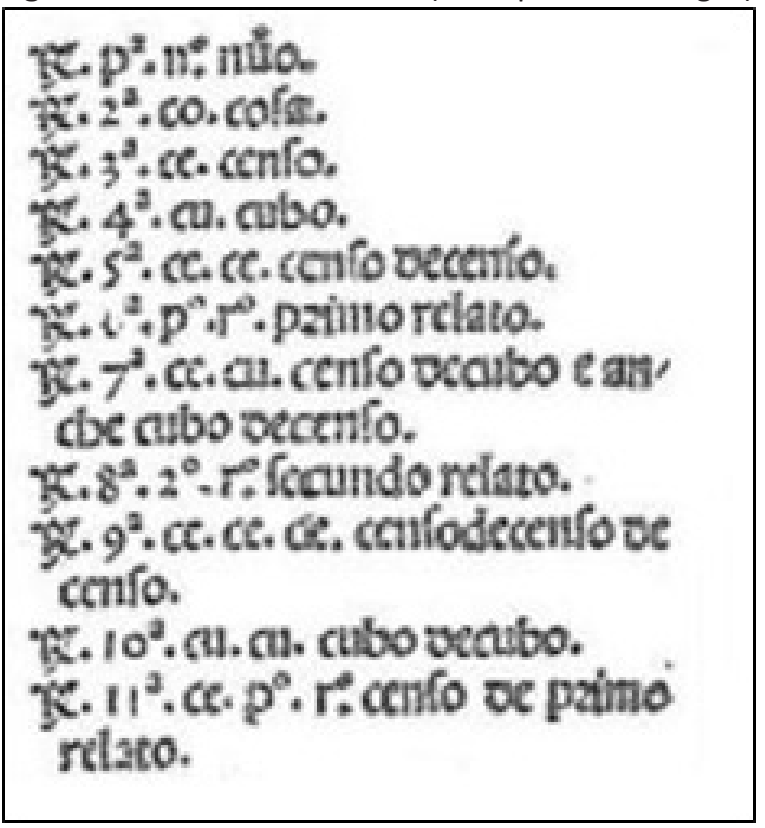

En esta misma línea, Cajori (1993, p. 108) recalca que:

The most commonly notations used by Luca Pacioli and by several later Italian writers of the sixteenth century employs for $x, x^{2}, x^{3}, x^{4}, x^{5}, x^{6}, x^{7}, \ldots$, the abbreviations co. (cosa), ce. (censo), cu. (cubo), ce.ce. (censo de censo), po.ro. (primo relato), ce.cu. (censo de cubo), $2^{\circ} . r^{\circ}$. (secundo relato), etc.,

esto es, las mismas abreviaturas que hemos documentado y analizado en los tres tratados del corpus del DICTER.

Por último, Núñez Salaciense emplea también, como puede observarse en el siguiente ejemplo, las abreviaturas más generalizadas: co. para cosa, ce. para censo y $c u$. para cubo, etc., procedentes de la corriente italiana:
Y en este exemplo pusimos la cosa ser 2, y conforme a este valor de la cosa veremos el valor de las otras dignidades y cómo suelen ser escriptas:

Figura 6. Notaciones Pedro Núñez Salaciense (1567, fol. $24 v$ )

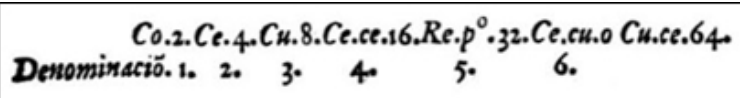

Al mismo tiempo, para la expresión de potencias más elevadas, presenta tablas en las que clasifica los valores de la potencia de la incógnita según una proporción tripla ( $v i d$. figuras 7 y 8 ), en la que se pone de manifiesto el mecanismo de multiplicar las distintas potencias:

Figuras 7 y 8 . Potencias de la incógnita o dignidades según tipo de proporción Pedro Núñez Salaciense (1567, fols. 27r-27v)

今્స Dignidad feptima relato fegūdo. ๙ิ Dignidad fexta cenfo de cubo. fं Dignidad quinta relato primo. $\dot{\infty}$ Dignidad quarta cenfo de cenfo. f. Dignidad tercera cubo. $\alpha$ Dignidad fegunda cenfo. $\therefore$ Dignidad primera cofa.

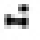

Dignidad .10. cenfo de relato primo. 59049. 9. cubo de cubo. 1968 ;.

8. cenfo de cenfo de cëfo. 6561. 7. relato fegundo. 2187. 6. cenfo de cubo. $\quad 729$. 5. relato primo. 243 . 4. cenfo de cenfo. 81 . 3. cubo.

2. cenfo.

1. cofa. 
Sin embargo, como hemos observado en el análisis de las dignidades (apartado 3.), «il semble ignorer presque complétement les algébristes allemands, Christoph Rudolf et Michel Stifel notamment lui sont inconnus» (Bosmans, 1907-1908, p. 157).

Este aspecto corrobora la tesis expuesta por Russo (1959, pp. 194-195), según la cual, «les simboles mathématiques, au $\mathrm{XVI}^{\mathrm{e}}$ siècle, varient sensiblement avec les auteurs", como a continuación, certifica:

Toutefois, ausein d'une mème école -allemande, italienne surtout - ils sont assez semblables et se présentent dans le temps selon des filiations assez logiques [...]. Les italiens sont de loin supérieus aux Allemands, qui, cependant, disposaient d'un bien meilleur système de notations.

De hecho, según Paradis y Malet, se deben a los alemanes «diferentes tentativas interesantes, aunque infructuosas, de elaborar una buena notación para la sucesión de potencias de la incógnita» (1989, p. 139), así como los vocablos sursólido y bisursólido, estudiados en los subapartados 3.5. y 3.7., expresados mediante los siguientes símbolos o abreviaturas en el Coss de Rudolff ${ }^{19}$, referencia directa de la Arithmética algebrática de Marco Aurel:

Figura 9. Notaciones Christoph Rudolff (1525, p. 174)

\section{9 oragmaodernumerus$$
22 \text { rabir }
$$$$
\text { z) jenfus }
$$$$
\text { ce cubus }
$$$$
\text { zक } 3 \mathrm{cn} \text { focjens }
$$$$
\beta \text { furfoliount }
$$$$
\text { zoce jenficubus. }
$$$$
\text { b?. biffurfolioum. }
$$$$
\text { zzzzenfjenposens }
$$$$
\text { cre cubur de cubo }
$$

Por otro lado, frente a la contribución y supremacía de los matemáticos y algebristas italianos, los de la escuela alemana introdujeron y popularizaron (fundamentalmente Michel Stifel, en su Arithmetica Integra, 1544) los símbolos $+y-{ }^{20}$; de tal manera que las aritméticas y álgebras alemanas del S. XVI habían desplazado los símbolos $p$ (abreviación del latín plus o del italiano più 'más') para la adición y $m$ (abreviación del latín minnus (íd.), DECH o del italiano meno) utilizados por los algebristas italianos. Estos aspectos se comprueban en los textos de nuestro corpus, en los que percibimos el contraste entre la obra del alemán Marco Aurel, que confirma:

fi podras:fino como en fummar fummafte conel + , aqui lo reftaras con $\mathrm{el}-$.

(1552, fol. 45r)

Por ejemplo, en la operación:

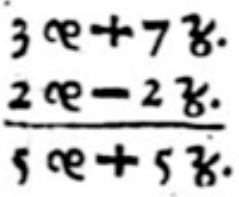

(1552, fol. 71r),

que en notación simbólica se expresaría:

$$
\begin{aligned}
& 3 x^{3}+7 x^{2} \\
& 2 x^{3}-2 x^{2} \\
& \hline 5 x^{3}+5 x^{2}
\end{aligned}
$$

Y la de Pérez de Moya, que defiende:

D'estos dos characteres $p$., $m$., notarás que la $p$. quiere dezir más y la m. menos; el uno es copulativo, el otro disiunctivo; sirven para summar y restar quantidades differentes, como adelante mejor entenderás (p. 453).

Como refleja la siguiente operación (p. 516):

\begin{tabular}{lllll}
4 & cv. & m. 2 & co. \\
3 & co. & m. & s & n. \\
\hline m. 20 & cv. & p. & 10. & co. \\
12 & cce. & m. & 6 & ce.
\end{tabular}

que, en la actualidad, se representaría mediante los siguientes símbolos:

$$
\begin{gathered}
4 x^{3}-2 x \\
3 x-5 \\
\hline-20 x^{3}+10 x \\
12 x^{4}-6 x^{2} \\
\hline-20 x^{3}+12 x^{4}-6 x^{2}+10 x
\end{gathered}
$$

O la empleada por Núñez Salaciense, quien, en la misma línea que Moya, se decanta por las abreviaturas italianas, al afirmar que: 
La palabra más se escribe assí: $\tilde{p}$ y, la palabra menos assí: $\tilde{m}$, y ternemos en la memoria que, aunque no se explique esta palabra más, como no se declarare que es menos, luego se entiende que es más.

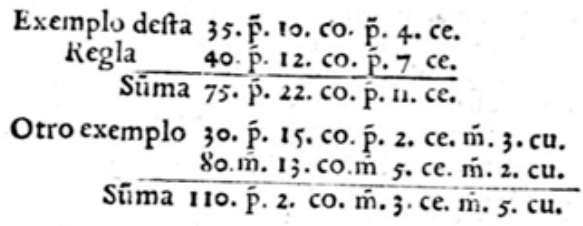

(Núñez, 1567, fol. 25r)

Operación de polinomios, que en notación o simbolismo actual, equivale a:

$$
\begin{array}{ll}
35+10 x+4 x^{2} & 30+15 x+2 x^{2}+3 x^{3} \\
40+12 x+7 x^{2} & 80-13 x-5 x^{2}-2 x^{3} \\
\hline 75+22 x+11 x^{2} & 110+2 x-3 x^{2}-5 x^{3}
\end{array}
$$

Por último, el signo para designar la 'igualdad', de manera análoga, se expresa mediante abreviaturas. Por ejemplo, Luca Pacioli emplea ae. (del latín aequālis, $D E C H$ ), aunque en este caso, a pesar de ser una referencia directa constante, no se documenta tal abreviatura en los textos examinados. Efectivamente, para la designación de la igualdad, hallamos la expresión igual $a-y$ su variante gráfica ygual $a-$, tanto en el Álgebra de Aurel, como en la de Núñez Salaciense:

Pongo el numero que fea ; 2 : multiplicadas en fi, vienc $9 \mathrm{z}$, ygual $2 ; 6 \mathrm{Q}$ :

(Aurel, 1552, fol. 76v)

\section{0.co.m்.1.ce. yguales a.26.}

(Núñez, 1567, fol. 22r)

O las abreviaturas ig. e yg. (de igual e ygual, ambas, a su vez, del latín aequālis, $D E C H$ ) en Pérez de Moya: «Esta figura ig. quiere decir igual» (p. 453). Una muestra de ello es la siguiente operación:

Pon que compró 1 co. de varas, la qual multiplica por 4 y será 4 co.; junta con ellas 21 y serán 4 co. $p$. 21.n, lo qual igualarás a 1ce., que son las varas que compró multiplicadas por sí, d'esta manera:

\section{4.co.p.zi.n.yg.à.1.ce.}

(Pérez de Moya, 1562, p. 595),

que en notación simbólica actual equivale a:

$$
4 x+21=1 x^{3} \text {. }
$$

En efecto, habrá que esperar al inglés Robert Recor$\mathrm{de}^{21}$ para la invención del signo $=$, que apareció por primera vez en su libro dedicado al álgebra, The Whetstone of Witte (publicado en 1557), donde justifica el autor el uso de esas dos líneas paralelas o rectas gemelas de una misma longitud; pues, explica Recorde, no hay dos cosas que puedan ser más iguales:

And to avoide the tediouse repetition of these words: "is equalle to", I will sette as I doe often in woorke use, a pair of paralleles, or Gemowe lines of one lengthe, thus: =======, because noe 2 thynges can be moare equalle.

Figura 10. Notación del signo igual Robert Recorde (Cajori, 1993, p. 165)

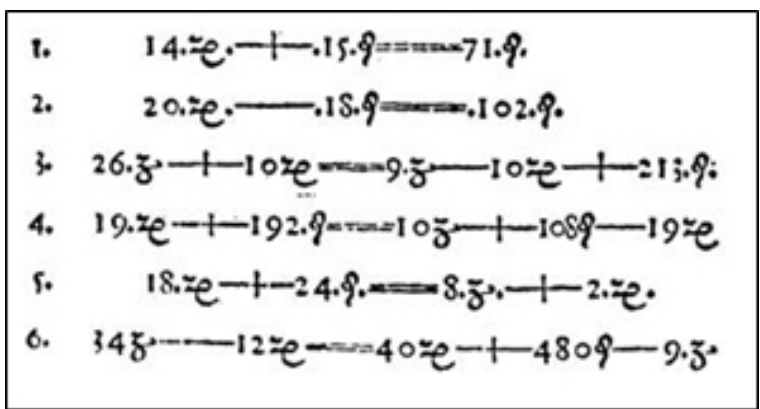

Expresiones algebraicas que se traducen al simbolismo moderno de la siguiente forma:

$$
\begin{aligned}
& \text { 1. } 14 x+15=71 \\
& \text { 2. } 20 x-18=102 \\
& \text { 3. } 26 x^{2}+10 x=9 x^{2}-10 x+213 \\
& \text { 4. } 19 x+192=10 x^{2}+108-19 x \\
& \text { 5. } 18 x+24=8 x^{2}+2 x \\
& \text { 6. } 34 x^{2}-12 x=40 x+408-9 x^{2}
\end{aligned}
$$

\section{CONCLUSIONES}

En primer lugar, en el análisis de las dignidades hemos constatado que estas designaciones y compuestos sintagmáticos - salvo el término cubo, referido a la tercera potencia: $x^{3}-$ no aparecen recogidos, con esta acepción matemática, en ninguno de los repertorios lexicográficos consultados, ni siquiera en los específicos; motivo por el que, para su definición, seguimos el patrón confeccionado por el DRAE para el término cubo, esto es, " $X$ potencia de un número o expresión algebraica, que se obtiene multiplicando estas cantidades $x$ veces por sí mismas" en todo el paradigma, que designa hasta 10 posibles potencias de la incógnita. 
En cuanto al origen de estos vocablos, destacamos un italianismo: cosa, referido a la primera potencia de la incógnita - cuyo valor es variable-, así como los sustantivos de origen latino: lado y raíz, del que son sinónimos, y los cultismos: censo y cubo, para la segunda y tercera potencia de la incógnita; consecuencia de las interesantes traducciones realizadas al latín por Roberto de Chester y Gerardo de Cremona (Cf. Hughes, 1986; Karpinski, 1915) de los arabismos shai', mâl y Kâb presentes en la obra de al-Khwārizmī, los cuales constituyen la base de las primeras notaciones del álgebra ${ }^{22}$.

A continuación, por lo que respecta al resto de las potencias, por un lado, tal y como hemos testimoniado en los ejemplos, y de acuerdo con la afirmación de Núñez Salaciense (1567, fol. 25v), hemos comprobado que «toda dignidad multiplicada en sý engendra otra de doblada denominación», mecanismo en el que las 3 dignidades elementales: cosa, censo y cubo, sirven de núcleo o base, pues, mediante su reduplicación o yuxtaposición, se generan potencias superiores, más elevadas. Este rasgo característico, según Cajori (1993), se documenta ya en las aritméticas árabes, en las que la mayoría de autores expresaban las potencias superiores según el principio multiplicativo (Kâb-mâl designaba $x^{6} ; K a ̂ b-K a ̂ b, x^{9}$ ) o el principio aditivo (Kâb-mâl designaba $x^{5} ; K a ̂ b-K a ̂ b, x^{6}$ ). Este hecho explica la aparición de los compuestos sintagmáticos: censo de cubo, $x^{6}\left(x^{2 \cdot 3=6}\right)$; cubo de cubo, $x^{9}\left(x^{3 \cdot 3=9}\right)$; censo (de) censo de censo, $x^{8}\left(x^{2 \cdot 2 \cdot 2=8}\right)$, formados mediante un principio multiplicativo, 0 censo de censo, $x^{4}\left(x^{2 \cdot 2=4}\right.$ y $\left.x^{2+2=4}\right)$, principio aditivo $y$ multiplicativo al mismo tiempo, estudiados.

No obstante, por otro lado, hallamos la confluencia de los compuestos cultos sursólido y bisursólido - acuñados por los cosistas alemanes - y los compuestos sintagmáticos primo relato o relato primero y relato segundo - utilizados por los algebristas italianos-, para la designación de la quinta y séptima potencia.

En cuanto al análisis de las abreviaturas, se comprueba que cada autor presenta una serie de tendencias o filiaciones. Por ejemplo, Marco Aurel, de origen germánico e introductor del álgebra en España, utiliza en su Libro primero de Arithmética algebrática (1552), tanto la nomenclatura como las grafías o abreviaturas diseñadas por los matemáticos alemanes más destacados e influyentes (entre otros, Rudolff, Ries, Stifel, etc.). Por el contrario, el portugués Núñez Salaciense ${ }^{23}$ se decanta por el esquema designativo establecido por los italianos Leonardo de Pisa y Luca Pacioli, fundamentalmente, así como por el uso de las síncopas empleadas por los mismos en sus tratados matemáticos. En contraposición, Pérez de Moya fusiona ambas tendencias en la nomenclatura, al conjugar tanto las designaciones de procedencia italiana como las alemanas, pero se desliga, sin embargo, de la escuela alemana en lo que respecta a las notaciones algebraicas de las potencias de la incógnita y los símbolos de las dos operaciones aritméticas básicas o elementales de adición y sustracción. En este sentido, como admite Rey Pastor (1926, p. 105), «su obra significa un retroceso».

En líneas generales, se puede afirmar que nos hallamos ante una "economía simbólica» o de "grafismos aceptados por convención» (Paradis y Malet, 1989, p. 84), imprescindibles en el desarrollo y evolución del álgebra en la península ibérica a lo largo del Quinientos.

En efecto, estos aspectos analizados ponen de manifiesto la importancia de los referentes, de la herencia cultural y del bagaje de conocimientos de las técnicas algebraicas anteriores a la creación del álgebra simbólica, marcada por la tradición de la geometría euclidiana ${ }^{24}$ que desembocará en un lenguaje preferentemente simbólico, en el que las ecuaciones predominan sobre las figuras, cuyo paso intermedio, semiverbal y semisimbólico al mismo tiempo, hemos mostrado.

En síntesis, de acuerdo con Mancho Duque ( $C f$. 2007a), "se constata un giro en el método de exposición matemático», que va de la retórica elemental a la madurez simbólica, esto es, de la palabra al símbolo. Proceso en el que, sin duda, la etapa y producción científica del Renacimiento, caracterizada por la proliferación de abreviaturas, contribuirá en gran medida tanto a la independización como a la consolidación y abstracción del álgebra ${ }^{25}$.

\section{AGRADECIMIENTOS}

Este trabajo se inserta en el marco del proyecto I+D+i: "El Diccionario de la Ciencia y de la Técnica del Renacimiento (DICTER): fases finales" (Ref.: FFI201016324/FILO) financiado por la Dirección General de Investigación del Ministerio de Ciencia e Innovación. Estas investigaciones se han podido llevar a cabo gracias a la ayuda predoctoral FPU, concedida por el Ministerio de Educación, Cultura y Deporte en 2011 (Ref.: AP2010-3663). 


\section{NOTAS}

1. Se conocen pocos datos sobre su biografía; de origen alemán, afincado en Valencia, fue maestro de escuela (1541) y publicó la primera obra impresa en España cuyos contenidos versan sobre la regla de la cosa o Álgebra, de ahí que en el prólogo exponga (1552: fol. IIIr): "es cosa nueva lo que trato y jamás vista ni declarada, y podrá ser que ni aun entendida ni imprimida en España" (Cf. Picatoste y Rodríguez, 1891; Rey Pastor, 1926; Paradis y Malet, 1989).

2. Matemático andaluz (Santisteban del Puerto, ca. 1513 - Granada, 1597), fue uno de los autores más célebres del panorama científico del S. XVI hispano debido a su labor divulgativa. Su obra alcanzó multitud de ediciones (unas 30, desde la fecha de su primera publicación en 1562 hasta 1875 ) y fue muy conocida dentro y fuera de nuestras fronteras (Cf. Picatoste y Rodríguez, 1891; Rey Pastor, 1926; Leal y Leal, 1971-1972; Valladares Reguero, 1997).

3. Cosmógrafo y matemático portugués (Alcácer do Sal, 1502 - Coimbra, 1578), es, junto a Pérez de Moya, otro de los autores más destacados del Quinientos (Cf. Picatoste y Rodríguez, 1891; Rey Pastor, 1926; Ventura Sousa, 1985). Su Libro de Álgebra, publicado 30 años antes en su lengua materna, portugués, tal y como explicita el autor en el prólogo (1567: fols. IIIr-IIIv), supuso un gran avance, ya que "al dedicar las dos primeras partes al Álgebra como tal confiere este a saber una entidad propia, que hasta entonces no se le había concedido. Si tenemos en cuenta que su libro fue escrito hacia 1537 podemos decir que se anticipó a Cardano, que es considerado el primero que dio autonomía al Álgebra en su obra: Ars Magna, publicada en el año 1545" (Flórez Miguel, 2006, p. 418), aspecto que Massa Esteve denomina como "la algebrització de les matemàtiques", esto es, "quan l'àlgebra comença a ser considerada una disciplina independent dins de la matemàtica" (2010, p. 101).

4. Accesible en la web del DICTER: http:// dicter.eusal.es/?idContent=elenco_obras.

5. Sobre las distintas designaciones del Álgebra en el Renacimiento hispano, léanse Mancho Duque, 2007a, 2007b; Vernet, 2006:185-187 y Molina Sangüesa, 2015c. A propósito de la consideración del Arte Mayor en la península ibérica del S. XVI, véase Massa Esteve (2012).
6. “Característica que se mantendrá a lo largo de los años por los matemáticos árabes, perdiéndose los pocos vestigios de tipo simbólico que se encuentran en la obra de Diofanto o en la obra de los hindúes" (Paradis y Malet, 1989, p. 50). Para más información léase Bell, 2000: 105-107. Sobre las abreviaturas o notaciones empleadas por Diofanto ( $C f$. Nesselmann, 1842 o Cajori, 1993, pp. 71-73).

\section{La cursiva de los ejemplos es nuestra.}

8. Destacan Paradis y Malet (1989, p. 68) la presencia de esta clasificación en obras árabes, como la de Al Aamoulí, en la que los autores "hacían la siguiente aclaración de la notación utilizada: La cantidad que se multiplica por ella misma se llama raíz en aritmética, lado en geometría y shai' (cosa) en álgebra; el resultado se llama entonces cuadrado".

9. Las palabras res y radix, para designar la incógnita, aparecen en la versión latina del Álgebra de al-Khwārizmī traducida por Roberto de Chester (Cf. Karpinski, 1915).

10. Sobre los conceptos "conjugar» y «conjugación» en el Libro de Álgebra de Pedro Núñez, léase Molina Sangüesa, 2014. En este problema, de construcción geométrica, cada uno de los lados desconocidos se pueden expresar como una raíz de una ecuación cuadrática con coeficientes numéricos conocidos, y esta raíz es, según Boyer (2003, p. 354), constructible geométricamente por medio de artificios conocidos de los Elementos de Euclides o del Álgebra de al-Khwārizmī, en el que cada uno de los lados se designan como "cosa" y entonces se resuelve el problema por la regla de la cosa y el "cuadrado", por medio de una ecuación de segundo grado.

11. Italiano de nacimiento (Cremona, 1114 - Toledo, 1187), fue uno de los traductores medievales más ilustres y prolíficos. A pesar de que se conserva poca información acerca de la vida de este autor (Cf. Boncompagni, 1851) se sabe, sin embargo, que conocía el griego, el árabe y el latín, y que sus traducciones abarcan una multiplicidad de temas (como la astronomía, geometría, óptica, matemáticas o la alquimia).

12. "Gerardo de Cremona tradujo māl por census, y no por quadratus, y esta traducción hizo tal fortuna que la palabra census, que en latín significa 'patri- monio', 'riqueza', fue usada en libros de álgebra escritos en latín en la época medieval, y también más adelante cuando en el Renacimiento empezaron a aparecer libros de álgebra en lenguas vernáculas. En estos, la palabra census, convertida en término técnico, cuyo significado en lenguaje natural ya carecía de importancia, no se tradujo sino que se castellanizó (censo), catalanizó (cens) o italianizó (censo)" (Puig, 2010, p. 90).

13. Más conocido como Fibonacci (hijo de Bonaccio, mercader y funcionario comercial) (Pisa, $1180-i$ ?, 1250). Viajó por negocios, y por placer, por toda Europa y el Cercano Oriente y fue el introductor de las cifras hindo-arábigas (Bell, 2000, p. 113). Autor que tomó como punto de referencia la obra de alKhwārizmī, pues en su Liber Abacci condensó los conocimientos aritméticos y algebraicos árabes y orientales, el cual ejerció una gran influencia en la etapa pre-renacentista (Paradis y Malet, 1989, pp. 93-99).

14. Luca Pacioli (Sansepolcro, 1445 Roma, 1517) fue uno de los autores más sobresalientes del Quattrocento italiano y es considerado como «il punto de partenza della matematica del Rinascimento» (Giusti y Maccagni, 1994, p. 15). Su obra, titulada: Suma de arithmetica, geometria, proportioni et proportionalità (1494), es la primera obra matemática impresa en lengua vernácula y el último de los tratados del ábaco. La Summa de Pacioli compiló todos los conocimientos de álgebra de los siglos anteriores - esto es, los contenidos de aritmética y álgebra del Liber Abacci-, en una sola obra de carácter enciclopédico, motivo por el que, según Martín Casalderrey (2000, p. 84), "se convirtió en la lectura básica para los algebristas del S. XVI, que, apoyados en ella, pudieron hacer nuevos descubrimientos".

15. "Of sursolidum Ries said that it was a "deaf number» (eine taube Zahl). In the manuscript of the Founder of Algebra, $\mathrm{x}^{5}$ is called surdumsolidum (deaf solid). The german taub and the Latin surdum are translations of the Arabic asam, used by the Arabs for the greek

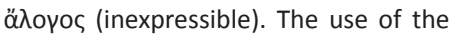
word solidum indicates that the cossists regarded certain powers as generalized cubes" (Bashmakova y Smirnova, 2000, pp. 64-65). 
16. "The most famous cossist was Adam Ries (Staffelstein, 1492 - Annaberg, 1559), who published many influential textbook on arithmetic. In 1525, he wrote a Coss manuscript which accurately reflected the state of algebra in his day" (Cf. Bashmakova y Smirnova, 2000).

17. Matemático germano (Silesia, 1499 Vienna, 1543), publicó el primer libro de álgebra escrito en alemán, el cual ejerció una gran influencia en la producción de otros matemáticos posteriores (Stifel, Aurel, etc.). Su contribución es relevante desde el punto de vista de la notación de las raíces (véase Molina Sangüesa, 2015d) y significativa por su clasificación y designación de las potencias de la incógnita.

18. Para mayor información sobre el simbolismo algebraico desarrollado por los distintos matemáticos de la Península ibérica, consúltense Docampo Rey (2008) y Romero Vallhonesta (2012).

19. "Rudolff's book Coss, written in 1525, is the first German algebra book [...]. Although this is the first German algebra book, containing some important innovations, there were already a number of algebra books (some only existing in manuscript) which Rudolff had studied before writing his text. These included

\section{REFERENCIAS BIBLIOGRÁFICAS}

\section{Fuentes primarias}

Aurel, M. (1552). Libro primero de Arithmética algebrática. Valencia: Joán de Mey.

Núñez Salaciense, P. (1567). Libro de Álgebra en Arithmética y Geometría. Anvers: Herederos de Arnoldo Birckman.

Pacioli, L. (1494). Suma de arithmetica, geometria, proportioni et proportionalità. Venezia: Paganino Paganini.

\section{Estudios}

Bashmakova, I. y Smirnova, G. (2000). The beginnings and evolution of Algebra. Washington: The Mathematical Association of America.

Bell, E. T. (2000). Historia de las matemáticas (5a edición). México: Fondo de Cultura Económica.

Boncompagni, B. (1851). Della vita e delle opere di Gherardo Cremonese, traduttore del secolo decimosecon-
Robert of Chester's translation of al-Khwarizmi's Hisab al-jabr w'al-muqabala, which was available in manuscript form, and he used a compilation of algebra texts by Johann Vögelin who worked in Vienna. Rudolff uses letters for algebraic quantities, unlike early algebra texts as he points out in Coss" [http://wwwhistory.mcs.st-and.ac.uk/Biographies/ Rudolff.html. Consulta: 13/12/13].

20. "The modern algebraic signs + and came into use in Germany during the last twenty years of the fifteenth century" (Cajori, 1993, p. 230). Fue Johann Widman el primero en utilizarlos en un libro impreso: una aritmética comercial, Rechenung auff allen Kauffmanschafft, publicada en 1489. Estos signos + y -, "que se utilizaban originalmente, al parecer, para indicar exceso y defecto en las medidas de mercancías en los almacenes, terminaron por pasar a ser símbolos para representar las dos operaciones aritméticas básicas de sumar y restar" (Cf. Boyer 2003, p. 360).

21. Matemático inglés (Tenby, Gales 1510 - ¿ं?, 1558), es considerado como el iniciador de la escuela matemática inglesa. Según Gutiérrez (2008, p. 89), "señala el despertar en su país de una matemática que llevaba dormida cerca de dos siglos, desde la muerte de Bradwardine".

Pacioli, L. (1509). De divina proportione. [Trad. y ed. Calatrava, J. (1991). La divina proporción. Madrid: Akal].

Pérez de Moya, J. (1562). Arithmética práctica y speculativa. Salamanca: Mathías Gast.

Pisa, L. (1202). Liber abacci.

Recorde, R. (1557). The Whetstone of Witte, which is the second part os Arithmetike,

do, e di Gherardo da Sabbioneta, astronomo del secolo decimoterzo. Atti dell'Accademia Pontificia dei Nuovi Lincei, 4, pp. 387-493.

Bosmans, H. (1907-1908). Sur le Libro de algebra de Pedro Nuñez. Bibliotheca Mathematica, 8, pp. 154-169.

Boyer, C. B. (2003). Historia de la matemática (2a edición). Madrid: Alianza Editorial.
22. Hemos estudiado estas cuestiones en Molina Sangüesa, en prensa.

23. En línea con la corriente italiana del pensamiento matemático, de la cual era un buen conocedor ( $C f$. Molina Sangüesa, $2015 a$ y $2015 b)$.

24. A propósito del cultivo de la geometría en los textos matemáticos renacentistas manejados en este estudio, véase Sánchez Martín (2009).

25. Y todo lo que ello conlleva en el desarrollo de otras disciplinas y del razonamiento matemático en general, pues afirma Bell (2000, p. 132) que "si no se hubiera transformado el álgebra elemental en una ciencia puramente simbólica a fines del siglo XVI, parece poco probable que la geometría analítica, el cálculo diferencial e integral, la teoría de probabilidades, la teoría de números y la dinámica pudieran haber arraigado y florecido, así como fue el caso, en el siglo XVII" e incluso llega a admitir que "la perfección del simbolismo algebraico fue una de las cosas que más contribuyó a la velocidad sin precedentes con que se desarrollaron las matemáticas".

containing the Extraction of root, the Cossike Practice, with the Rules of Equation, and the Woorkes of Surde Numbers. London.

Ries, A. (1525). Coss.

Rudolff, C. (1525). Behend und Hübsch Rechnung Durch die Kunstreichen Regeln Algebra, so Gemeincklich die Coss Genennt Werden. Staßburg.

Cajori, F. (1993). A History of Mathematical Notations. 2 vols. La Salle (Illinois): Open Court Publishing Co., reprinted by Dover.

Corominas, J. y Pascual, J. A. (1980-1991). Diccionario Crítico Etimológico Castellano e Hispánico. Madrid: Gredos.

Couchoud, S. (1993). Mathématiques Egyptiennes. Recherches sur les connaisances mathématiques d'Egypte pharaonique. París: Éditions Le Léopard d’Or. 
Docampo Rey, J. (2008). Vernacular algebra in the Iberian Peninsula before Marco Aurel: Notations and terminology. En Hunger, H., Seebacher, F. y Holzer, G. (eds.). Proceedings of the 3rd International Conference of the European Society for the History of Science. Viena, pp. 85-92.

Etayo Miqueo, J. J. (1986). El álgebra del cinquecento. Historia de la Matemática hasta el siglo XVII. Madrid: Real Academia de Ciencias Exactas, Físicas y Naturales, pp. 147-169.

Flórez Miguel, C. (2006). Ciencias, siglos XVXVII. En Rodríguez-San Pedro Bezares, L. E. (coord.). Historia de la Universidad de Salamanca (vol. 3). Salamanca: Ediciones Universidad de Salamanca, pp. 409-443.

Fowler, D. y Robson, E. (1998). Square Root Approximations in Old Babylonian Mathematics: YBC 7289 in context. Historia Mathematica, 25, pp. 366-378.

Giusti, E. y Maccagni, C. (1994). Luca Pacioli e la matematica del Rinascimento. Firenze: Editorial Giunti.

Glare, P. G. W. (1968-1982). Oxford Latin Dictionary. Oxford: Clarendon Press.

Gutiérrez, S. (2008) Robert Recorde: el creador del signo igual. Suma, 57, pp. 89-95.

Hughes, B. (ed.) (1986). Gerard of Cremona's Translation of al-Khwārizmī's Al-jabr: A Critical Edition". Mediaeval Studies, 48, pp. 211-263.

Karpinski, L. C. (ed., trad.) (1915). Robert of Chester Latin transalation of the algebra of al-Khowarizmi. London: MacmiIlan Company.

Leal y Leal, L. (1971-1972). El Bachiller Juan Pérez de Moya. Boletín del Instituto de Estudios Giennenses, 70-71, pp. 17-36.

Mancho Duque, M.a J. (2005). La divulgación científica y técnica en castellano en la época de Cervantes. En Becedas, M., Flórez, C. y Mancho Duque, M.a J. (eds.). La Ciencia y la Técnica en la época de Cervantes. Salamanca: Publicaciones Universidad de Salamanca, pp. 17-51.

Mancho Duque, M. a J. (2007a). Oriente y occidente en el léxico de las matemáticas del Quinientos. En Campos, M. Cotelo, R. y Pérez Pascual, J. I. (eds.). Historia del léxico español. Anexos Revista de Lexicografía, 5, pp. 97-107.

Mancho Duque, M.a J. (2007b) Aproximación al léxico matemático del Re- nacimiento. En Delgado, I. y Puigvert, A. (eds.). Ex admiratione et amicitia. Homenaje a Ramón Santiago. Madrid: Ediciones del Orto, pp. 723-740.

Mancho Duque, M.a J. (dir.) (2015). Diccionario de la Ciencia y de la Técnica del Renacimiento (DICTER). Salamanca: Ediciones Universidad de Salamanca. [En línea]. Disponible en http://dicter. eusal.es.

Martín Casalderrey, F. (2000). Cardano y Tartaglia. Las matemáticas en el Renacimiento italiano. Madrid: Nivola.

Massa Esteve, M. a R. (2010). Àlgebra i Geometría al Libro de Álgebra en Arithmética y Geometría (1567) de Pedro Núñez. Quaderns d'Història de l'Enginyeria, 11 pp. 101-129.

Massa Esteve, M.a R. (2012). Spanish Arte Mayor in the Sixteenth century. En Rommevaux, S., Spiesser, M. y Massa Esteve, M. a R. (dirs.). Pluralité de l'Algèbre à la Renaissance. París: Honoré Champion Éditeur, pp. 103-126.

Molina Sangüesa, I. (2014). Cruce entre gramática y matemática: los conceptos de "conjugar» y "conjugación» en el $\mathrm{Li}$ bro de Álgebra en Arithmética y Geometría (1567) de Pedro Núñez Salaciense. En Marcos de Dios, A. (ed.). La lengua portuguesa. Estudios lingüísticos. Salamanca: Ediciones Universidad de Salamanca, pp. 505-517.

Molina Sangüesa, I. (2015a). Tradición e innovación en el ámbito de la divulgación matemática de Quinientos. En Blume, J. y López Ferrero, C. (eds.). La ciencia como diálogo entre teorías, textos y lenguas. Berlín: Frank \& Timme, pp. 31-48.

Molina Sangüesa, I. (2015b). Las matemáticas en el Renacimiento hispano: estudio léxico y glosario [Tesis Doctoral Inédita]. Universidad de Salamanca: Salamanca.

Molina Sangüesa, I. (2015c). Glosario de aritmética y álgebra en el Renacimiento hispano. En Mancho, M.a J. (dir.). Diccionario de la Ciencia y de la Técnica del Renacimiento (DICTER). Salamanca: Ediciones Universidad de Salamanca. [En línea]. Disponible en http://dicter.usal. es/?idContent=matematicas.

Molina Sangüesa, I. (2015d). En torno a las designaciones de raíz y sus notaciones: una muestra de la consolidación del álgebra sincopada en el Renacimiento hispano. Verba. Anuario Galego de Filoloxía, 42, pp. 323-346.
Molina Sangüesa, I. (en prensa). El legado de al-Khwārizmī: análisis de la traducción e introducción de algunos arabismos en el campo del álgebra hispánica renacentista. Quaderns: revista de traducció. Barcelona: Universitat Autònoma de Barcelona.

Nesselman, G. H. F. (1842). Versucheiner Kritischen Geschichte der Algebra, 1. Teil. Die Algebra der Griechen. Berlin: G. Reimer.

Paradis, J. y Malet, A. (1989). Los orígenes del álgebra: de los árabes al Renacimiento. Barcelona: Promociones y Publicaciones Universitarias.

Picatoste y Rodríguez, F. (1891). Apuntes para una biblioteca científica española del siglo XVI. Madrid: Imprenta y Fundación Manuel Tello.

Puig, L. (2010). Historias de al-Khwārizmī. El proyecto algebraico. Suma, 65, pp. 87-94.

Real Academia Española (2001). Diccionario de la lengua española (22a edición). Madrid: Espasa Calpe. [En línea] Disponible en http://buscon.rae.es/diccionario/drae.htm.

Rey Pastor, J. (1926). Los matemáticos españoles del siglo XVI. Madrid: Biblioteca Scientia.

Romero Vallhonesta, F. (2012). Algebraic symbolism in the first algebraic works in the Iberian Peninsula. Philosophica, 87, pp. 117-152

Russo, F. (1959). La constitution de l'algèbre au XVIe siècle. Étude de la structure d'une évolution. Revue d'histoire des sciences et leur applications, 12, 3, pp. 193-208.

Sánchez Martín, F. J. (2009). Estudio del léxico de la geometría aplicada a la técnica en el Renacimiento hispano. Salamanca: Ediciones Universidad de Salamanca.

Valladares Reguero, A. (1997). El Bachiller Juan Pérez de Moya: Apuntes biobibliográficos. Boletín del Instituto de Estudios Giennenses, 165, pp. 371-412.

Ventura Sousa, M. (1985). Vida e Obra de Pedro Nunes. Lisboa: Instituto de Cultura e Língua Portuguesa, Ministério da Educação.

Vernet, J. (2006). Lo que Europa debe al Islam de España (2a edición). Barcelona: Acantilado. 